\title{
EVERY FINITELY GENERATED FISCHER GROUP IS FINITE
}

THEODORE S. BOLIS

\begin{abstract}
By a simple combinatorial argument, and using a result of Bruck, the Burnside problem is settled affirmatively for the class of Fischer 3-groups.
\end{abstract}

0. Introduction. In a recent book on cubic forms by Yu. I. Manin [1] the Burnside problem for the class of Fischer 3-groups was posed (cf. p. 39 of [1]). In this note we give a simple combinatorial argument leading to an affirmative settlement of this problem. A result of H. R. Bruck [2] that every finitely generated commutative Moufang loop of period 3 is finite, is essential to our proof. We also need some theorems connecting symmetric quasigroups, Moufang loops and Fischer groups.

1. Definitions. A Fischer group is a pair $(G, E)$ such that (i) $G$ is a group, (ii) $E$ is a generating subset of $G$, and (iii) $x^{2}=1,(x y)^{3}=1$ and $x y x \in E$ for all $x, y \in E$.

It is easy to see that $E$ is a complete conjugacy class of $G$ and that the commutator subgroup $G^{\prime}$ is generated by the elements of the form $x y$, $(x, y \in E)$. If we define the following operation on $E$

$$
x \circ y=x y x
$$

then $(E, \circ)$ becomes a distributive symmetric quasigroup, i.e. we have $x \circ y=y \circ x, x \circ(x \circ y)=y, x \circ(y \circ z)=(x \circ y) \circ(x \circ z) \quad(x, y, z \in E)$. Moreover if we fix $u \in E$ and define

$$
x * y=u \circ(x \circ y), \quad x, y \in E,
$$

we make $(E, *)$ a commutative Moufang loop, i.e. all the axioms for an abelian group hold except associativity, which is replaced by weak associativity

$$
(x * y) *(x * z)=x^{2} *(y * z) \quad\left(x, y, z \in E, x^{2}=x * x\right) .
$$

2. Some auxiliary results. We mention here some results needed in the proof of our main theorem.

Received by the editors April 23, 1973.

AMS (MOS) subject classifications (1970). Primary 20F10; Secondary $20 \mathrm{~N} 05$.

Key words and phrases. Fischer group, distributive symmetric quasigroup, commutative Moufang loop.

(c) American Mathematical Society 1974 
2.1 THEOREM (BRUCK [2]). Every finitely generated commutative Moufang loop of period 3 (i.e. $x^{3}=1$ if $x$ is an element of the loop) is finite.

2.2 Proposition (Benkov-Belousov [4]). If $E$ is a distributive symmetric quasigroup and $u \in E$ is fixed, then $(E, *)$ is a commutative Moufang loop of period 3 with identity element $u$, where $x * y=u \circ(x \circ y)$. Conversely, if $E$ is a commutative Moufang loop of period 3 and $c$ is in the associative center of $E(i . e . c *(x * y)=(c * x) * y$ for all $x, y \in E)$, then $(E, \circ)$ is $a$ distributive symmetric quasigroup where $x \circ y=c * x^{-1} * y^{-1}$.

2.3 Proposition (CF. [1, Theorem 3.8]). Let $(G, E)$ be a Fischer group and let $(E, \circ)$ be the distributive symmetric quasigroup with operation defined by (1). If $T(E)$ denotes the group generated by the permutations $\left(t_{x}\right)_{x \in E}, t_{x}(y)=x \circ y$, then the map $f: E \rightarrow T(E)$ defined by $x \rightarrow t_{x}$ can be extended to $G$ as a group homomorphism and $\operatorname{ker} f=Z(G)=$ the center of $G$. Thus $(T(E), E)$ is a Fischer group and $T(E) \cong G / Z(G)$. Here we identify $x \in E$ with $t_{x} \in T(E)$.

3. Statement of the problem. We say that a set $I$ generates a Fischer group $(G, E)$ if $I \subset E$ and $I$ generates $G$. If $I$ is finite, is $G$ finite? Let us construct the free Fischer group generated by $I$. Let $F(I)$ be the free group generated by $I, N$ the normal subgroup of $F(I)$ generated by the words $x^{2},\left(s_{1} x s_{1}^{-1} s_{2} y s_{2}^{-1}\right)^{3}, x, y \in I, s_{1}, s_{2} \in F(I), G(I)=F(I) / N, f: F(I) \rightarrow G(I)$ (the canonical map) and $E(I) \subset G(I)$ the conjugacy class containing $f(I)$. Then $(G(I), E(I))$ is the free Fischer group generated by $I$ and $E(I)$ the free distributive symmetric quasigroup generated by $I$. By using 2.1 and 2.2 we see that $E(I)$ is finite if $I$ is finite. But unfortunately 2.3 tells us only that $G(I) / Z(G(I))$ is finite.

4. We now proceed to establishing our result. We start with the following combinatorial.

4.1 Proposition. Let $(G, E)$ be a Fischer group with $\# E=n$. Then

$$
\begin{aligned}
\# G & \leqq \sum_{k=0}^{m} \prod_{i=1}^{k}\left(n-\frac{1}{2} i(i-1)\right) \\
& =1+n+n(n-1)+\cdots+\prod_{i=1}^{m}\left(n-\frac{1}{2} i(i-1)\right)
\end{aligned}
$$

where $m=\max \left\{k \in N: \frac{1}{2} k(k-1) \leqq n\right\}$.

Proof. Let $W_{k}$ denote the irreducible words of length $k$ with letters in $E$. Then $\# W_{0}=1, \# W_{1}=n$. We claim that

$$
\# W_{k} \leqq \prod_{i=1}^{k}\left(n-\frac{1}{2} i(i-1)\right) \quad \text { if } \frac{1}{2} k(k-1) \leqq n, \quad k=1,2, \cdots
$$


Indeed, let $x_{1} x_{2} x_{3} \cdots x_{k} \in W_{k}$. Then for $x_{1}$ there are $n$ possible choices, for $x_{2}$ there are $n-1$ possible choices $\left(x_{2} \neq x_{1}\right.$, because otherwise the length of the word could be reduced by 2 since $x^{2}=1$ ), for $x_{3}$ we have $n-3$ possible choices $\left(x_{3} \neq x_{1}, x_{2}\right.$, and $\left.x_{1} x_{2} x_{1}\right)$, for $x_{4}, n-6$ choices $\left(x_{4} \neq x_{1}, x_{2}, x_{3}, x_{1} x_{2} x_{1}, x_{1} x_{3} x_{1}, x_{2} x_{3} x_{2}\right)$ etc. Thus the total number of words with irreducible length $k$ is at most $\prod_{i=1}^{k}\left(n-\frac{1}{2} i(i-1)\right)$. If $k \geqq 2$ this is a pretty liberal estimate, e.g. if $x y x=z \in E$, then $x y=y z=z x$ etc.

4.2 THEOREM. Every finitely generated Fischer group is finite.

Proof. Suppose that the group is generated by a finite set $I$. It is enough to prove that the free Fischer group $(G(I), E(I))$ is finite. By Proposition 2.2 and Theorem 2.1, $E(I)$ is finite. Therefore $G(I)$ is finite by Proposition 4.1.

5. Remarks. Fischer groups were defined in [3] in a more general way involving a general prime $p$ instead of 3. By a theorem due to Fischer (under finiteness assumption) it is obtained that $\# E=3^{k}, \# G=2 \cdot 3^{m}$ and $G$ is solvable. It would be interesting to know the exact order of $G$ if we know that $\# E=3^{k}$ and no other relations are introduced except those warranted by the definition of a Fischer group. For example it is easy to see that $\# W_{2}=3^{k-1}\left(3^{k}-1\right)$.

\section{REFERENCES}

1. Yu. I. Manin, Kubičeskie formy (Cubic forms), "Nauka", Moscow, 1972.

2. H. R. Bruck, A survey of binary systems, Springer-Verlag, New York, 1966.

3. B. Fischer, Distributive Quasigruppen endlicher Ordnung, Math Z. 83 (1964), 267-303. MR 28 \#4055.

4. V. D. Belousov, The structure of distributive quasigroups, Mat. Sb. 50 (92) (1960), 267-298. (Russian) MR 22 \#11059.

Department of Mathematics, State University College, Oneonta, New York 13820 